\title{
Conformation of Adsorbed Layers of polyNIPAM on Silica in a Binary Solvent
}

\author{
Shannon M. Notley* \\ Department of Applied Mathematics, Research School of Physical Sciences and Engineering, Australian \\ National University, Canberra 0200 ACT, Australia
}

Received: July 3, 2008; Revised Manuscript Received: July 24, 2008

\begin{abstract}
The conformation of poly( $N$-isopropylacrylamide) chains adsorbed at a silica interface was studied as a function of concentration in the methanol-water binary solvent mixture. Both water and methanol are good solvents for PNIPAM; however, in certain mixtures cononsolvency is induced by a lowering of the LCST. This led to a decrease in the extent of the PNIPAM layer away from the interface as measured using the colloidal probe technique in the poor solvent region. At low methanol concentrations but still in the good solvent region capillary bridging between the silica surfaces with adsorbed PNIPAM layers was observed due to the increased methanol concentration in this interfacial region over that of the bulk. Furthermore, adsorption measurements showed that PNIPAM adsorbed only weakly to the silica interface with a low surface excess on the order of $0.23 \mathrm{mg} / \mathrm{m}^{2}$, which allowed study of the behavior of the immobilized PNIPAM chains under highly dilute conditions using the quartz crystal microbalance. As the concentration of methanol increased toward the phase transition boundary, a slight contraction followed by an expansion of the PNIPAM was observed, which is in agreement with previous predictions from theory for polymers in solution.
\end{abstract}

\section{Introduction}

The adsorption of polymers and polyelectrolytes at an interface has been a research focus in many branches of science including chemistry, physics, and biology as well as in more applied areas such as chemical engineering for many decades due in large part to the many technological and natural applications where adsorbed layers of this "soft" matter may highly influence the energy of interaction of these hence modified surfaces. ${ }^{1}$ Adsorbed polymers are routinely used to control adhesion and lubrication as well as impart stability to colloidal dispersions or alternately cause aggregation and flocculation. ${ }^{2,3}$ Technologies reliant upon modification of surface properties by adsorbed polymer and polyelectrolyte layers include minerals processing, wastewater treatment, and paper making to name but a few. ${ }^{4}$

Steric stabilization of colloidal dispersions continues to be an area of research interest although many advances have been made in the past 50 years. ${ }^{5}$ In order to stabilize a particle against aggregation, the adsorbed polymer must contribute a significant repulsive force such that the close approach of the colloidal particles is unfavored. Hence, the adsorbed layer conformation is of the utmost importance. That is, extension of the polymer away from the interface into the solvent phase must dominate over other apparent surface forces such as those due to electrostatics or the ubiquitous van der Waals forces. The repulsive forces due to overlap of polymer layers develops due to the increased concentration of polymer in the gap between the surfaces over that in the bulk, giving rise to an osmotic pressure. Furthermore, the restricted movement of polymer chains in the overlap region is also entropically unfavorable. Hence, stable dispersions may be achieved through cognisant control of the extension of the polymer layer away from the interface.

* To whom correspondence should be addressed. E-mail: shannon.notley@ anu.edu.au.
There have been many studies over the past two decades investigating the adsorption of polymers, both charged and uncharged, at the solid-liquid interface and the subsequent effect on the forces of interaction between colloidal particles. ${ }^{6-17}$ Adsorption of uncharged polymers to a charged interface tends to be weak in the absence of the strong driving force of ionic attraction. Many surface forces studies have focused on welldefined, grafted homopolymer layers in order to compare to the available theories on overlapping of polymer layers. ${ }^{18-21}$ In this study the thermo-responsive polymer poly $(N$-isopropylacrylamide) (PNIPAM) has been used as the freely adsorbed homopolymer. PNIPAM has been well-studied previously, particularly when looking at the phase transition from good to poor solvency when moving through the lower critical solution temperature (LCST) which in water is $32{ }^{\circ} \mathrm{C} .{ }^{22-26}$ The LCST of PNIPAM is influenced by a number of factors including $\mathrm{pH}$, ionic strength, and the presence of alcohol and is retained if the PNIPAM molecules are grafted or freely adsorbed at the solid-liquid interface.

The behavior of PNIPAM in binary mixtures of water and methanol has been studied in solution but not adsorbed at an interface to the knowledge of the author. This system is of interest for numerous reasons. Methanol and water are both good solvents for PNIPAM; however, cononsolvency can be induced for certain concentrations of the slightly better solvent methanol. Winnik et al. studied the solvency of PNIPAM in the binary solvent system as a function of methanol concentration and showed that the LCST reached a minimum at a mole fraction of 0.35 and at that mole fractions greater than 0.45 no LCST could be observed. ${ }^{27}$ Thus, the methanol concentration in the binary solvent system may be used to probe effects near this critical point of solvency, especially as concentration may be more easily controlled than temperature. Recent studies, both theoretical and experimental, have suggested that rich conformational behavior of polymers exists near the critical point in binary solvent systems for extremely dilute conditions. ${ }^{28-33}$ Brochard and de Gennes predicted such behavior almost 30 
years ago. ${ }^{34}$ The better solvent, in this case methanol, condenses around the PNIPAM chains, creating a cloud of good solvent, thereby attracting other polymer segments and contributing to a long-range attractive force to other monomers in the chain, resulting in a reduction in size. It is hence the goal of the present study to investigate if such effects can also be observed for adsorbed polymer layers and, in particular, for PNIPAM in the binary solvent mixture of methanol and water.

\section{Experimental Section}

Materials. Poly( $N$-isopropylacrylamide) was synthesized according to the method of Zhou et al. ${ }^{35}$ The polymer was twice precipitated from an acetone/hexane mixture with purity confirmed using ${ }^{13} \mathrm{C}$ NMR. The molecular weight was determined using GPC to be $9.0 \times 10^{5}$ Da with low polydispersity.

Oxidized silicon wafers from Peregrine Semiconductor Australia (Homebush Bay, Australia) were used for adsorption measurements using optical reflectometry as well as the surface forces measurements. These silica surfaces had a $305 \mathrm{~nm}$ oxide layer thickness measured using ellipsometry (Beaglehole Instruments, New Zealand).

The polymer solutions were prepared in Milli-Q water. The methanol used in this study for preparation of binary solvent systems with water was of AR grade and purchased from Sigma (Australia).

Methods. Adsorption of PNIPAM to Silica. PNIPAM was dissolved in Milli-Q water for use in the force measurements as well as the adsorption experiments involving the optical reflectometer (OR) and quartz crystal microbalance (QCM-D). PNIPAM was adsorbed to the silica surfaces used in these measurements from a 100 ppm solution in Milli-Q water. Prior to adsorption, all silica surfaces were cleaned using a $10 \% \mathrm{w} / \mathrm{w}$ aqueous solution of $\mathrm{NaOH}$ followed by rinsing in water and ethanol. Finally, the silica surfaces were given a moderate water plasma treatment to ensure that the silica surface is fully hydroxylated to promote adsorption of PNIPAM through $\mathrm{H}$ bonding.

Both OR and QCM-D were used to determine the surface excess and adsorption kinetics of the PNIPAM to silica. In $\mathrm{OR},{ }^{36,37}$ an oxidized silica wafer with defined oxide layer thickness is used as the substrate. An incident laser beam is passed through a $45^{\circ}$ prism onto this reflective silica surface. The laser beam is reflected back through another $45^{\circ}$ prism into the detection system where the parallel (p) and perpendicular (s) components are measured. Changes in the optical properties due to an adsorbing species will alter the ratio of the $\mathrm{p}$ and $\mathrm{s}$ components. This ratio can be converted to a surface excess using a four-layer optical model (silicon:silica:adsorbing species: solvent) if the refractive indices and layer thicknesses are accurately known. Using this technique, adsorption of PNIPAM to silica is performed under well-defined hydrodynamic conditions with the incident laser beam striking the surface at the stagnation point with a constant flow of polymer solution supplied to the liquid cell. Thus, adsorption is diffusion limited. Hence, OR gives valuable information regarding the surface excess and adsorption kinetics.

QCM-D was also used to monitor adsorption of PNIPAM to a silica-coated quartz crystal. A KSV QCM Z500 (KSV, Finland) was used with a $5 \mathrm{MHz}$ AT-cut quartz crystal (KSV, Finland) as the adsorbing surface. The resonance frequency along with the third, fifth, and seventh overtones were continuously monitored as a function of time. The sensed mass of the crystal is proportional to the decrease in resonance frequency according to the Sauerbrey equation. ${ }^{38}$ This sensed mass will include the effective "dry mass" of polymer adsorbed to the surface as well as a proportion of solvent which is entrained within the polymer layer up to the plane of shear. Furthermore, the resonance frequency of the crystal will be influenced by the properties of the fluid above the interfacial region as demonstrated by Kanazawa. ${ }^{39}$ The QCM-D was used to measure the adsorbed amount of PNIPAM to silica in water and can be compared to the data gathered using OR to determine the proportion of solvent within the layer. ${ }^{40}$

The QCM-D can also be used to determine the viscoelastic properties of adsorbed layers as well as approximate the layer thickness. ${ }^{41,42}$ The energy dissipated by the adsorbed layer can be determined by monitoring the impedance of the crystal as a function of adsorption which may be modeled if data from multiple overtones is considered. ${ }^{43}$ Thus, qualitative information on polymer layer structure due to changes in solvent properties can be deduced using QCM-D.

All adsorption measurements were carried out at $23^{\circ} \mathrm{C}$.

Colloidal Probe Microscopy (CPM). The interaction between silica surfaces with adsorbed PNIPAM layers in the binary solvent system was measured using CPM. In this technique, a silica sphere of $10 \mu \mathrm{m}$ radius was attached to the end of an atomic force microscope cantilever with a small amount of adhesive according to the method of Ducker et al. ${ }^{44}$ Standard, contact $\mathrm{Si}_{3} \mathrm{~N}_{4}$ cantilevers (Veeco, USA) were used for all force measurements in this study. The data were typically collected using the $200 \mu \mathrm{m}$ long, triangular-shaped cantilever with a spring constant of $0.29 \mathrm{~N} / \mathrm{m}$ as measured by the thermal noise method. ${ }^{45}$ The typical surface forces measurement using this technique has been described extensively elsewhere. ${ }^{46}$ The measured forces were normalized by the probe radius in order to compare between experiments as well as convert the forces to the potential energy of interaction through application of the Derjaguin approximation. ${ }^{47}$ All surface forces measurements were taken in polymer-free solution at a temperature of $23{ }^{\circ} \mathrm{C}$.

Theories of Overlapping Adsorbed Polymer Layers. The normalized force as a function of surface separation can be compared to various theories dealing with compression of polymer layers at an interface. In order to accurately compare the measured surface forces data to these theories, the segment density profile of the adsorbed polymer layer away from the solid-liquid interface must be known. Very few techniques are able to determine this segment density profile accurately with neutron reflectivity one notable example. However, the apparent forces on approach for two surfaces bearing adsorbed layers may be fit to available theories sufficiently well to provide information on the extent of the interfacial polymer layer. The two most often used theories are the Alexander-de Gennes theory ${ }^{18,48}(\mathrm{~A}-\mathrm{dG})$ and the Milner-Witten-Cates theory ${ }^{20}$ (MWC). The $\mathrm{A}-\mathrm{dG}$ theory assumes a step profile for the adsorbed layer, while the MWC theory uses a more realistic parabolic segment density profile. Both of these theories were strictly developed for end-terminated polymer brush layers with high grafting densities, but previous studies have shown reasonable agreement between experiment and theory for freely adsorbed homopolymer or polyelectrolyte layers. ${ }^{12,16,40}$ The normalized force may be fit using eqs $1(A-d G)$ and 2 (MWC) to determine the layer thickness, $L_{0}$, and the grafting density, $\Gamma$. 


$$
\begin{gathered}
F_{r}=\frac{16 \pi}{35} k_{\mathrm{B}} T L_{0} \Gamma^{3 / 2}\left[12-7\left(\frac{D}{2 L_{0}}\right)^{-5 / 4}-5\left(\frac{D}{2 L_{0}}\right)^{7 / 4}\right] \\
F_{r}=4 \pi P_{0}\left[\frac{2 L_{0}^{*}}{D}+\left(\frac{D}{2 L_{0} *}\right)^{2}-\frac{1}{5}\left(\frac{D}{2 L_{0} *}\right)^{5}-\frac{9}{5}\right]
\end{gathered}
$$

with

$$
P_{0}=\frac{k_{\mathrm{B}} T N}{2}\left(\frac{\pi^{2} l^{4}}{12}\right)^{1 / 3} \Gamma^{5 / 3}
$$

where $N$ is the number of polymer segments of length $l$.

It is important to note that the MWC theory was developed for the interaction between two impenetrable brushes and not for a polymer brush confined by a hard wall. A simple correction factor exists, namely, $L_{0}=1.34 L_{0} *$, to correlate the equilibrium brush thicknesses derived from both the $\mathrm{A}-\mathrm{dG}$ and MWC theories. $^{12}$

In the collapsed state the adsorbed globular-like PNIPAM chains may be expected to behave like so-called "mushrooms". Under these conditions the measured force-distance profiles may be fit with the Dolan and Edwards theory (DE) for interacting surfaces with polymers adsorbed in such a conformation. $^{21}$ The DE theory is given by eq 3 , where $\Gamma$ is the grafting density and $R_{\mathrm{g}}$ can be replaced by $R_{\mathrm{h}}$ if the polymer is in a good solvent.

$$
F / r \approx\left(\frac{72 \pi k_{\mathrm{B}} T}{\Gamma}\right) e^{-D / R_{\mathrm{g}}}
$$

\section{Results and Discussion}

The surface forces on approach between two PNIPAM-coated silica surfaces will be influenced by a number of experimentally variable factors including, specifically in this study, the properties of the solvent system. To be able to fully understand these forces it is important to measure the adsorbed amount of PNIPAM to the silica surface as this will provide a basis for fitting of the interaction data to theory through knowledge of the packing of polymer chains at the interface. Figure 1 shows the adsorption of PNIPAM to silica in water from a solution with a concentration of $100 \mathrm{ppm}$ measured using OR. The surface excess rapidly reaches an equilibrium value of $0.23 \mathrm{mg} /$ $\mathrm{m}^{2}$ after about $10 \mathrm{~min}$. This corresponds to a packing density of 1 chain per $6500 \mathrm{~nm}^{2}$. The PNIPAM is irreversibly adsorbed to the silica surface through $\mathrm{H}$ bonding, and as there is little to no ionic interaction between the polymer and surface and, furthermore, as the polymer is in a good solvent, the adsorbed amount is low.

The adsorption of PNIPAM to silica was also determined using QCM-D. With this technique the mass of polymer and solvent entrained within the layer is measured. Hence, by comparing to the OR data, the proportion of solvent within the adsorbed layer can be determined. Figure 2 shows the "sensed" mass as a function of adsorption of PNIPAM as well as the change in energy dissipation of the resonating crystal as a result of the adsorbed layer with time. Interestingly, in comparison to the OR data no equilibrium is reached in the QCM experiment even after $1 \mathrm{~h}$. In the QCM material is not continually flowing to the surface, rather it is done under "batch" conditions. It is reasonable to assume that the major part of the adsorption process is rapidly achieved as indicated by the kinetics; however, there is some continued adsorption or layer rearrangement as a function of time, the latter of which is supported by the surface forces data to be presented in this study. Thus, if the value of the surface excess (determined from the sensed mass using the Sauerbrey equation) in this rapid adsorption regime, measured

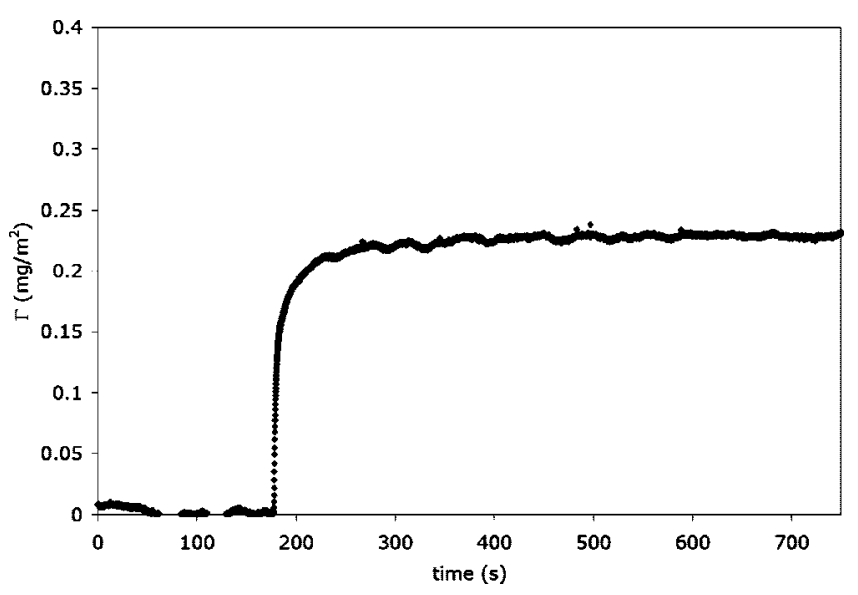

Figure 1. Adsorption of PNIPAM to a silica surface studied using optical reflectometry. The adsorbed amount rapidly reaches equilibrium after $10 \mathrm{~min}$. The polymer concentration was $100 \mathrm{ppm}$ and adsorbed from Milli-Q water at $23{ }^{\circ} \mathrm{C}$.

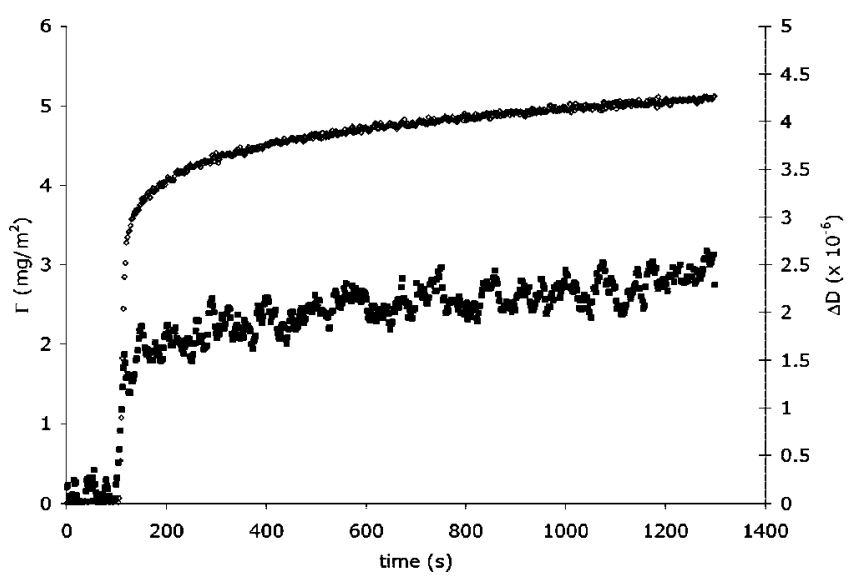

Figure 2. QCM-D data for adsorption of PNIPAM to the silica-coated quartz crystal from a solution in water with a concentration of 100 ppm. Sensed mass (open symbols) and energy dissipated (closed symbols) shown.

to be of the order of $5 \mathrm{mg} / 2$, is compared to the OR data, then the proportion of solvent entrained within the layer is very high, near $95 \%$. This is reasonable as water is a good solvent for PNIPAM, so it may be expected that the polymersolvent interactions will be maximized in comparison to polymer-surface interactions.

The presence of a highly extended polymer layer is supported by the corresponding energy dissipation data measured in parallel to the change in adsorbed mass using the QCM-D technique. There is a significant increase in the energy dissipated as a function of adsorption, which suggests a rather loosely packed, expanded polymer brush-like adsorbed layer conformation at this interface. Furthermore, by considering the frequency and impedance shifts due to adsorption of PNIPAM to the silicacoated quartz crystal at multiple overtones, an estimate of the layer thickness may be obtained using the model developed by Johannsman. ${ }^{43}$ Analyzing the data in this way resulted in a layer thickness of between 5 and $6 \mathrm{~nm}$, where the density of the interfacial layer used was the arithmetic mean of the polymer density and that of water for the ratio $1: 19\left(\sim 0.98 \mathrm{~g} / \mathrm{cm}^{3}\right)$. This value reflects only the initial adsorption of polymer to the interface (during the first $10-15 \mathrm{~min}$ ) and not the equilibrium thickness some $24 \mathrm{~h}$ later.

QCM-D was also used to investigate changes in the adsorbed layer structure of PNIPAM as a function of concentration of 


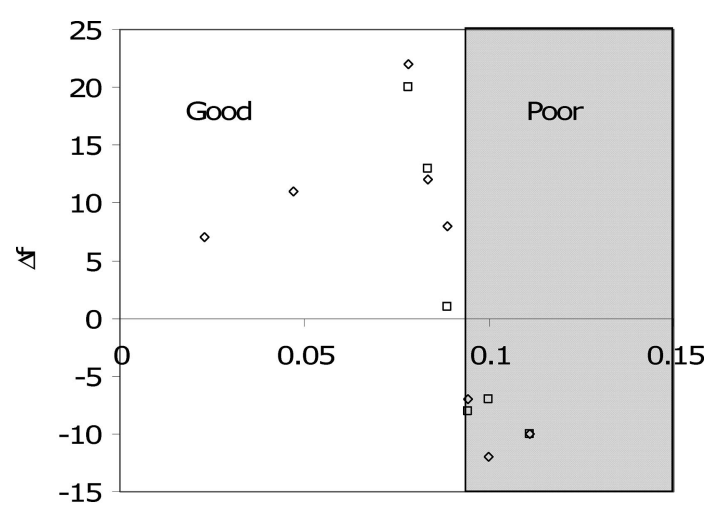

methanol mole fraction

Figure 3. Change in frequency and energy dissipation as a function of methanol mole fraction of the adsorbed PNIPAM layer on the silicacoated quartz crystal. Dashed line indicates the change in resonance frequency due to changes in the fluid properties. (Open symbols) $\Delta f_{3}$ for two different runs. The boundary between good and poor solvent conditions at $23{ }^{\circ} \mathrm{C}$ is at a methanol mole fraction of $0.09 .{ }^{27}$

methanol in water in the binary solvent system studied here. The analysis is complicated by the changes in the fluid properties above the sensing crystal as well as the preferential adsorption of the better solvent, methanol, to the polymer-rich interface. Changes in the resonance frequency of the crystal due to changes in the viscosity and density of the fluid are easily accounted for and may simply be factored out. ${ }^{39}$ However, the methanolwater ratio in the adsorbed layer remains unknown, and as such, the data presented here is only discussed in a qualitative fashion. Figure 3 shows the change in resonance frequency as a function of methanol concentration for the adsorbed PNIPAM layer. In the good solvent region an increase in methanol concentration results in a slight increase in the frequency. A maximum in the frequency is observed just prior to the phase boundary at 23 ${ }^{\circ} \mathrm{C}$, which corresponds to a methanol mole fraction of 0.09 . At methanol concentrations in the poor solvent region the change in frequency is negative, indicating a greater mass coupled to the surface. These results perhaps seem counterintuitive insofar as a collapse of the adsorbed layer would release bound solvent, thereby resulting in an increase in resonance frequency. However, a previous study by Ishida and Biggs showed similar results for the phase transition of grafted PNIPAM layers achieved through increasing temperature. ${ }^{49}$ In that study the authors suggested that the grafting density plays an important role and is in agreement with a previous study. ${ }^{50}$ For high graft density layers no such transition was observed with the QCM-D technique; ${ }^{26}$ however, Ishida and Biggs argued that for lowdensity grafted layers the increased molecular freedom allows the chains to collapse toward the interface. This then allows the polymer molecule to more effectively couple to the crystal under the oscillatory motion, thereby registering as an increasing "sensed" mass.

A recent study by Grabowski and Mukhopadhyay ${ }^{31}$ provides an alternative explanation for the QCM-D data in Figure 3. They studied the contraction and reswelling of poly(acrylic acid) near the critical point in a binary liquid mixture of lutidine-water. A slight decrease in the size of single chains of PAA in dilute solutions was observed as the critical point temperature became nearer. However, very close to the critical point the polymer chains increased in size significantly above that when the critical temperature was far off. The same effects are seen here in this study demonstrated with the QCM-D in Figure 3 for a very low density adsorbed layer of PNIPAM. This collapse and reswelling phenomena has long been predicted by theory for

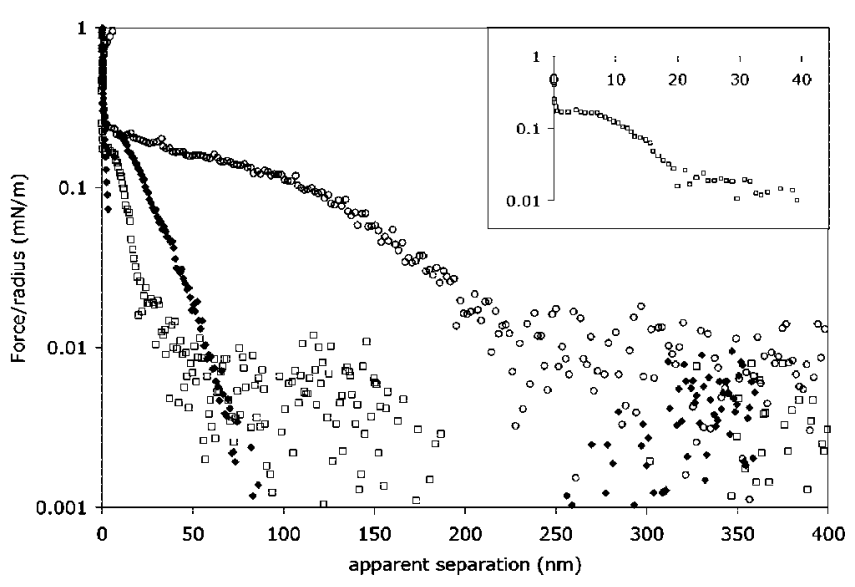

Figure 4. Forces on approach for silica surfaces in water in the absence and presence of adsorbed PNIPAM layers. Closed diamonds are in the absence of PNIPAM, open squares are PNIPAM layers after $1 \mathrm{~h}$, and open circles are PNIPAM layers after $24 \mathrm{~h}$. All force measurements are in polymer-free solution. (Inset) Data after $1 \mathrm{~h}$ highlighting the steric repulsive force curve.

polymer chains near the critical point in a binary liquid of two good solvents under extremely dilute conditions. ${ }^{34}$ Figure 3 shows that this contraction and reswelling phenomena is not restricted to polymer in solution but also polymers adsorbed at an interface as well.

The surface forces between silica surfaces with adsorbed layers of PNIPAM were also studied here using the colloidal probe technique. In this technique the apparent forces were measured in polymer-free solution as a function of methanol concentration. Figure 4 shows the forces on approach in water as a function of time. As can be seen in Figure 4, after only $1 \mathrm{~h}$ the surface forces profile change from the purely DLVO interaction $^{44,51,52}$ as expected for two clean silica surfaces interacting in aqueous solution to a small steric repulsive force with addition of PNIPAM equivalent to an adsorbed layer extent of approximately $8 \mathrm{~nm}$ per surface. It is interesting to note, though, that the range of the steric interaction force increases significantly if measured after $24 \mathrm{~h}$ of equilibration in water only. This supports the QCM-D data that suggested the reconformation of the PNIPAM chains at the interface and not the continued adsorption.

After the polymer layers were equilibrated in water and the surface forces measured, the concentration of methanol in the liquid cell was increased. Figure 5 shows the repulsive forces apparent as a function of methanol concentration. For all methanol mole fractions shown here the surface forces on approach are characterized by a significant steric repulsive force due to overlap of the PNIPAM chains at close separations. The extent of this repulsive force is at a minimum in the methanol mole fraction range of $0.09-0.42$ where, at the temperature for which the measurements are undertaken, that being $23{ }^{\circ} \mathrm{C}$, the PNIPAM chains are in poor solvent conditions. ${ }^{27}$ Therefore, collapse of the polymer layer toward the interface in this methanol concentration region as directly measured by the surface forces technique is not surprising, and similar behavior has been observed for grafted PNIPAM layers in water above the LCST ${ }^{49}$ It is noteworthy however that the re-entrant phase transition back to good solvent conditions at methanol mole fractions above 0.45 , resulting in the reswelling of the adsorbed layer, is also observed for these effectively immobilized PNIPAM chains. This transition has previously been only observed for the polymer in solution and not at the interface. ${ }^{53}$ Furthermore, the assertion that methanol is a slightly better 


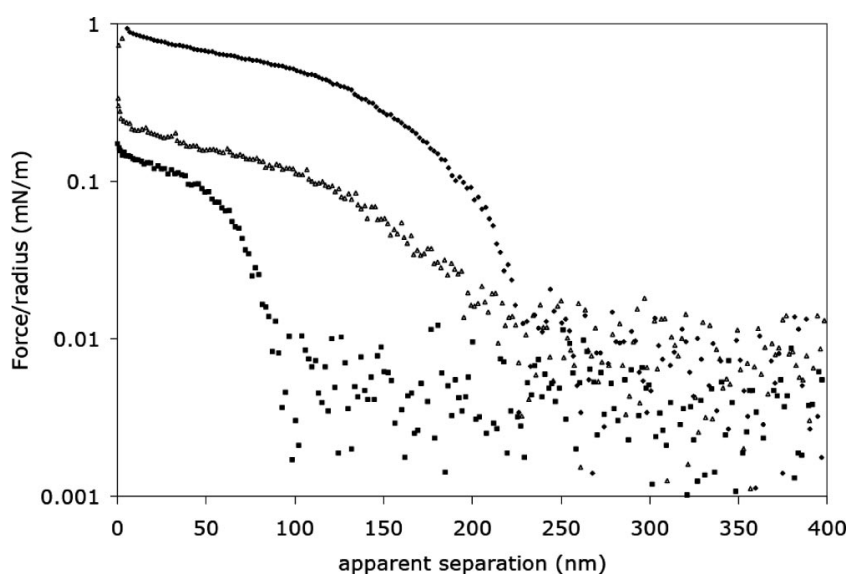

Figure 5. Forces on approach for silica surfaces with adsorbed PNIPAM as a function of methanol concentration. Open diamonds in pure methanol, open triangles in pure water, and closed squares in methanol with a mole fraction of 0.23 .

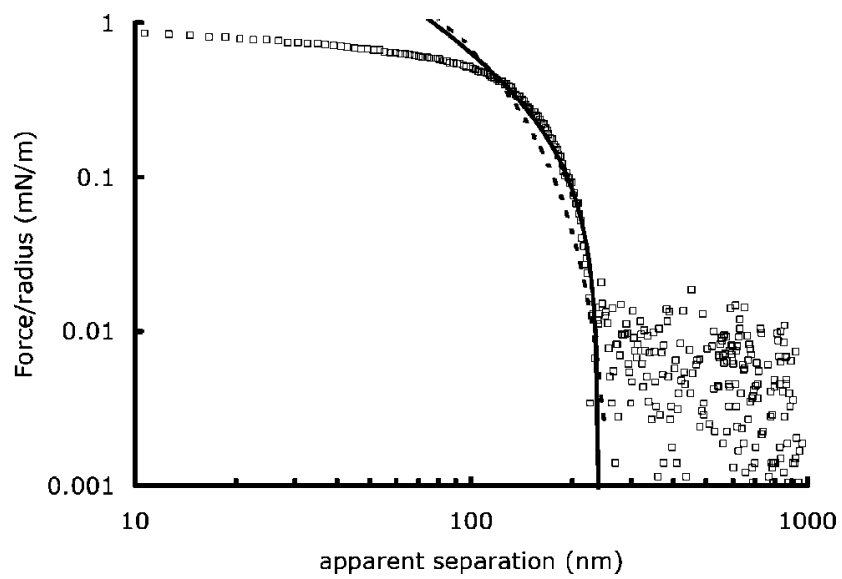

Figure 6. Fits to the Alexander-de Gennes theory (solid line) and Milner-Witten-Cates theory (dashed line) for the interaction between adsorbed PNIPAM layers in methanol. All force measurements are in polymer-free solution. An off set of $40 \mathrm{~nm}$ was used.

solvent than water is supported here with these surface forces measurements, which shows that the extent of the PNIPAM is far greater.

An attempt was made to fit the surface forces data to available theories concerning overlap of adsorbed polymer layers as outlined previously in this paper. Figure 6 shows the best fits to the $\mathrm{A}-\mathrm{dG}$ and MWC theories for the PNIPAM layer in pure methanol. In this case, a significant steric layer is apparent; however, the grafting density, that is the area occupied by a PNIPAM chain, is rather low as determined from the adsorption data using OR. At intermediate to large surface separations, the measured steric forces can be reasonably fit to both the $A-d G$ and MWC theories if an off set (by substituting $\mathrm{D}+\mathrm{D}_{0}$ for $\mathrm{D}$ ) is applied. Neither theory holds particularly well at short surface separations, which may be due to a number of factors such as the grafting density being too low to use these theories and the effective "splaying" of polymer chains due to compression of the colloidal probe. ${ }^{54,55}$

Under some circumstances the forces on approach are characterized by a steeply repulsive force followed by a longrange attraction and then another repulsive force presumably of steric origin. Typically, such force interaction curves as shown in Figure 7 were only seen for mole fractions of methanol in the range of $0.02-0.05$ corresponding to effectively good solvent conditions but with a decreased LCST over that of

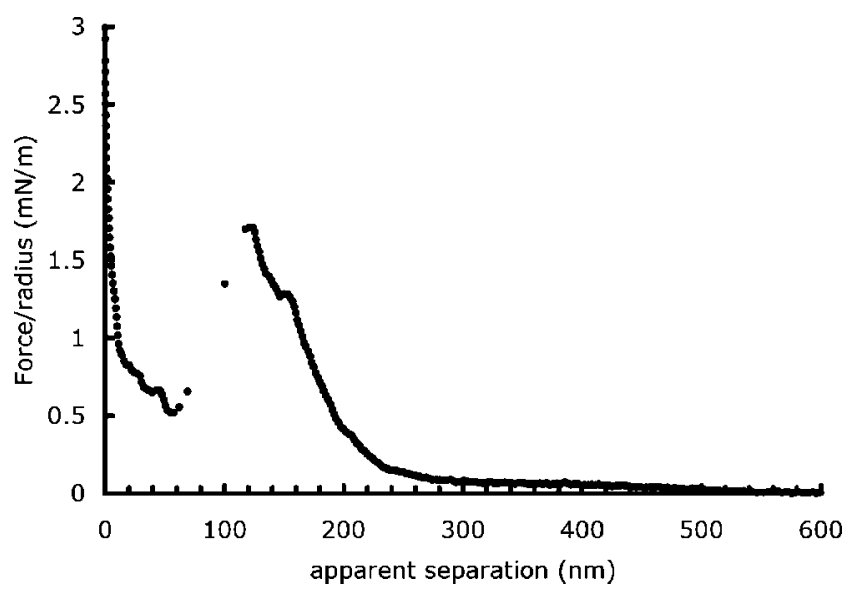

Figure 7. Forces on approach of PNIPAM-coated silica surfaces in a binary solvent system demonstrating the long-range attractive force. The methanol mole fraction is 0.05 .

PNIPAM chains in pure water. In the binary solvent system where methanol is a slightly better solvent than water methanol is condensed around the PNIPAM chains. This leads to an increased concentration of methanol in the interfacial regions over that in the bulk when the polymers are adsorbed to the silica surfaces. The data in Figure 7 suggests that at low polymer overlap separations when bulk solvent is beginning to be excluded from the gap the methanol-rich interfacial layers are capable of forming a capillary bridge between the probe and surface, effectively raising the concentration of methanol even further in the gap, resulting in a shift to a lower still LCST. This results in collapse of the PNIPAM chains toward the solid-liquid interface, giving rise to the attractive forces observed in Figure 7 at surface separations of 50-120 nm.

\section{Conclusions}

Adsorption of PNIPAM to silica in water was studied using OR and QCM-D. The adsorbed amount was relatively low, as expected due to the relatively weak interaction between PNIPAM and silica through hydrogen bonding. The adsorbed layer contained a significant amount of entrained water, indicating a very loose packing of chains at the interface. Surface forces measurements also showed that a significant steric layer developed extending about $100 \mathrm{~nm}$ away from the interface per surface. QCM-D measurements also indicated collapse followed by expansion of the polymer layer upon approaching the critical point in the methanol-water binary solvent system. This is in agreement with the theory that methanol condenses at low concentrations around the PNIPAM chains contributing to a long-range attractive force between monomer segments. This was supported by surface forces measurements where capillary bridging was observed for low methanol concentrations, resulting in collapse of the PNIPAM layer toward the interface. At higher methanol concentrations the surface forces data showed that the extent of the layer decreased in the poor solvent region; however, the layer once again expanded in pure methanol to a greater degree than in pure water, in agreement with the greater solvophilicity of methanol over for water for PNIPAM.

Acknowledgment. Funding from the Co-operative Research Centre for Functional Communication Surfaces (CRC SmartPrint) is gratefully acknowledged. Also, Tim Senden and Vince Craig, ANU, are acknowledged for continued support, guidance, and helpful discussions. Shaun Howard is also acknowledged for help with the optical reflectometry. 


\section{References and Notes}

(1) Fleer, G. J.; Cohen Stuart, M. A.; Scheutjens, J. M. H. M.; Cosgrove, T.; Vincent, B. Polymers at interfaces; Chapman and Hall: New York, 1993. (2) Hunter, R. J. Foundations of Colloid Science;Clarendon Press: Oxford, 1989.

(3) Israelachvili, J. Intermolecular and surface forces, 2nd ed.; Academic Press: New York, 1991.

(4) Napper, D. H. Polymeric Stabilization of Colloidal Dispersions; Academic Press: New York, 1983.

(5) Netz, R. R.; Andelman, D. Phys. Rep. 2003, 380, 1-95.

(6) Klein, J.; Luckham, P. F. Nature 1984, 308, 836.

(7) Hadziioannou, G.; Patel, S.; Granick, S.; Tirrell, M. J. Am. Chem. Soc. 1986, 108, 2869-2876. 3421.

(8) Biggs, S.; Healy, T. J. Chem. Soc., Faraday Trans. 1994, 90, 3415-

(9) Claesson, P. M.; Dahlgren, M. A. G.; Eriksson, L. Colloids Surf. 1994, A 93, 293.

(10) Biggs, S. Langmuir 1995, 11, 156-162.

(11) Yamamoto, S.; Ejaz, M.; Tsujii, Y.; Matsumoto, M.; Fukuda, T. Macromolecules 2000, 33, 5602-5607.

(12) Eframova, N. V.; Bondurant, B.; O’Brien, D. F.; Leckband, D. E. Biochemistry 2000, 39, 3441-3451.

(13) Notley, S. M.; Biggs, S.; Craig, V. S. J. Macromolecules 2003, 36 (8), 2903-2906.

(14) Valle-Delgado, J. J.; Molina-Bolivar, J. A.; Galisteo-Gonzalez, F.; Galvez-Ruiz, M. J.; Feiler, A.; Rutland, M. W. J. Phys. Chem. B 2004, 108 (17), 5365-5371.

(15) Claesson, P. M.; Poptoshev, E.; Blomberg, E.; Dedinaite, A. Adv. Colloid Interface Sci. 2005, 114-116, 173-187.

(16) McLean, S. C.; Lioe, H.; Meagher, L.; Craig, V. S. J.; Gee, M. L. Langmuir 2005, 21, 2199-2208.

(17) Wang, J.; Butt, H. J. J. Phys. Chem. B 2008, 112, 2001-2007.

(18) Alexander, S. J. Phys. (Paris) 1977, 38, 983.

(19) de Gennes, P. G. Adv. Colloid Interface Sci. 1987, 27, 189.

(20) Milner, S. T.; Witten, T. A.; Cates, M. E. Macromolecules 1988, $21,2610-2619$.

(21) Dolan, A. K.; Edwards, S. F. Proc. R. Soc. London A 1974, 337, 509-516.

(22) Haupt, B. J.; Senden, T. J.; Sevick, E. M. Langmuir 2002, 18, 2174 2182.

(23) Ishida, N.; Kobayashi, M. J. Colloid Interface Sci. 2006, 297, 513519

(24) Kidoaki, S.; Ohya, S.; Nakayama, Y.; Matsuda, T. Langmuir 2001, 17, 2402-2407.

(25) Liu, G.; Cheng, H.; Yan, L.; Zhang, G. J. Phys. Chem. B 2005, $109,22603-22607$.
(26) Liu, G.; Zhang, G. J. Phys. Chem. B 2005, 109, 743-747.

(27) Winnik, F. M.; Ringsdorf, H.; Venzmer, J. Macromolecules 1990, $23,2415-2416$.

(28) To, K.; Choi, H. J. Phys. Rev. Lett. 1998, 80, 536-539.

(29) Morita, S.; Tsunomori, F.; Ushiki, H. Eur. Polym. J. 2002, 38, $1863-$ 1870

(30) Castellanos, P.; Norman, A. I.; Greer, S. C. J. Phys. Chem. B 2006, 110, 22172-22177.

(31) Grabowski, C. A.; Mukhopadhyay, A. Phys. Rev. Lett. 2007, 98 , 207801.

(32) Sumi, T.; Kobayashi, K.; Sekino, H. J. Chem. Phys. 2007, 127, 164904.

(33) Dua, A.; Vilgis, T. A. Macromolecules 2007, 40, 6765-6769.

(34) Brochard, F.; de Gennes, P. G. Ferroelectrics 1980, 30, 33.

(35) Zhou, S.; Fan, S.; Au-Yeung, S. C. F. Polymer 1995, 36, 1341.

(36) Dijt, J. C.; Cohen Stuart, M. A.; Hofman, J. E.; Fleer, G. J. Colloids Surf. 1990, 51, 141-158.

(37) Dijt, J. C. Ph.D. Thesis, Landbouwuniversiteit te Wageningen, 1993.

(38) Sauerbrey, G. Z. Phys. 1959, 155, 206.

(39) Kanazawa, K. K.; Gordon, J. G. Anal. Chim. Acta 1985, 175, 99.

(40) Notley, S. M.; Biggs, S.; Craig, V. S. J.; Wågberg, L. Phys. Chem. Chem. Phys. 2004, 6, 2379-2386.

(41) Rodahl, M.; Höök, F.; Krozer, A.; Brzezinski, P.; Kasemo, B. Rev. Sci. Instrum. 1995, 66, 3924-3930.

(42) Notley, S. M.; Eriksson, M.; Wågberg, L. J. Colloid Interface Sci. 2005, 292, 29-37.

(43) Johannsmann, D. Macromol. Chem. Phys. 1999, 200, 501-516.

(44) Ducker, W. A.; Senden, T. J.; Pashley, R. M. Nature 1991, 353, 239.

(45) Hutter, J. L.; Bechhoefer, J. Rev. Sci. Instrum. 1993, 64, 18681873.

(46) Senden, T. J. Curr. Opin. Colloid Interface Sci. 2001, 6, 95.

(47) Derjaguin, B. V. Z. Kolloid 1934, 69, 155.

(48) de Gennes, P.-G. Macromolecules 1981, 14, 1637-1644.

(49) Ishida, N.; Biggs, S. Langmuir 2007, 23, 11083-11088.

(50) Plunkett, K. N.; Zhu, X.; Moore, J. S.; Leckband, D. E. Langmuir 2006, 22, 4259-4266.

(51) Ducker, W. A.; Senden, T. J.; Pashley, R. M. Langmuir 1992, 8, $1831-1836$.

(52) Hartley, P. G.; Larson, I.; Scales, P. J. Langmuir 1997, 13, 22072214.

(53) Zhang, G.; Wu, C. J. Am. Chem. Soc. 2001, 123, 1376-1380.

(54) Murat, M.; Grest, G. S. Macromolecules 1996, 29, 8282.

(55) Guffond, M. C.; Williams, D. R. M.; Sevick, E. M. Langmuir 1997, 13,5691 .

JP805842B 